\title{
Sensitivity analysis of critical experiments with evaluated nuclear data libraries
}

\author{
D. Fujiwara ${ }^{\mathrm{a}}$ and S. Kosaka
}

Nuclear Engineering Dept., TEPCO SYSTEMS CORPORATION, 2-37-28 Eitai Koto-ku, Tokyo 135-0034, Japan

\begin{abstract}
Criticality benchmark testing was performed with evaluated nuclear data libraries for thermal, lowenriched uranium fuel rod applications. C/E values for $k_{e f f}$ were calculated with the continuous-energy Monte Carlo code MVP2 and its libraries generated from ENDF/B-VI.8, ENDF/B-VII.0, JENDL-3.3 and JEFF-3.1. Subsequently, the observed $k_{\text {eff }}$ discrepancies between libraries were decomposed to specify the source of difference in the nuclear data libraries using sensitivity analysis technique. The obtained sensitivity profiles are also utilized to estimate the representativity of cold critical experiments to the boiling water reactor under hot operating condition.
\end{abstract}

\section{Introduction}

The evaluated nuclear data library ENDF/B-VII.0 was released in December 2006. From the view point of the thermal, low-enriched uranium fuel rod benchmark calculations, the main revised data from ENDF/B-VI.8 library are as follows [1]: the ${ }^{238} \mathrm{U}$ capture cross section in thermal and resonance range, ${ }^{16} \mathrm{O}(\mathrm{n}, \alpha)$ cross section in fast region and ${ }^{1} \mathrm{H}$ thermal scattering kernel.

This work is intended to compare the accuracy of criticality prediction with evaluated nuclear data libraries ENDF/BVI.8, ENDF/B-VII.0, JENDL-3.3, and JEFF-3.1 for Boiling Water Reactor (BWR) applications. The criticality benchmark testing was performed using the existing critical experiments conducted at Toshiba's Nuclear Critical Assembly (NCA) facility. The discrepancy of the $k_{\text {eff }}$ values between libraries were decomposed with sensitivity analyses to understand the physical mechanism caused by the difference of nuclear data. Finally, the representativity of the critical experiments to the application system of BWR in hot operating condition is assessed with sensitivity analysis to confirm the validity of this benchmark testing.

\section{Critical experiments}

Series of critical experiments at NCA were applied for the criticality benchmark testing. NCA is a tank-type light water moderated criticality facility and its criticality is adjusted by water level. Figure 1 shows the geometrical configuration of NCA core loading, which were arranged to simulate BWR 9x9-type fuel. The core consists of inner $2 \times 2$ assemblies test region and outer driver region containing $2 \mathrm{wt} \%$ enriched $\mathrm{UO}_{2}$ fuel pins. All the experiments were performed at room temperature with full density light water. Reduced water density under hot operating condition was simulated with insertion of aluminum tubes in the test region. The diameter of the aluminum tubes were adjusted for $0 \%$ and $40 \%$ void ratio. Table 1 shows the arrangement of the test region in each

\footnotetext{
${ }^{a}$ Presenting author, e-mail: fujiwara-daisuke@tepsys.co.jp
}

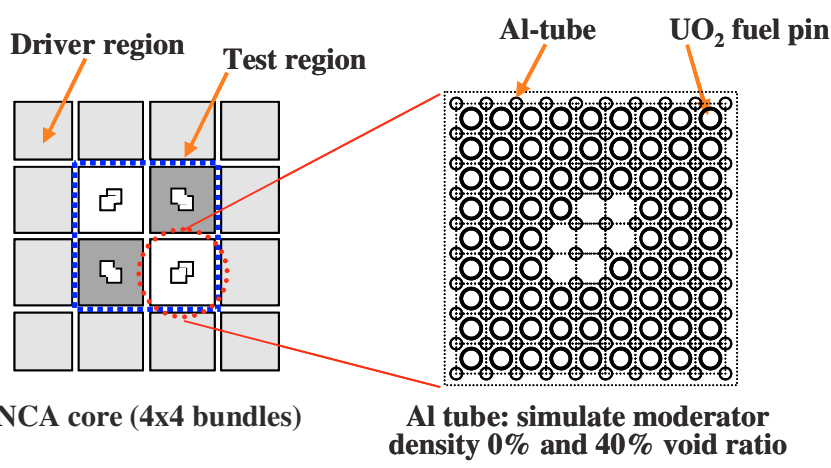

Fig. 1. Geometric configuration of NCA Core loading.

Table 1. Fuel pin and aluminium tube arrangements of test region for each experiment.

\begin{tabular}{ll}
\hline \multirow{2}{*}{ Fuel pin arrangement } & $\begin{array}{l}\text { Al-tube arrangement } \\
\text { H/HM (void ratio) }\end{array}$ \\
\hline \multirow{2}{*}{$\begin{array}{l}\text { 2 wt\% uniform enrichment } \\
\text { core }\end{array}$} & $6.9(0 \%)$ \\
\cline { 2 - 2 } & $7.2(0 \%, 40 \%)$ \\
\hline $\begin{array}{l}\text { 1 wt } \% \text { and 3wt } \% \\
\text { enrichment mismatch core }\end{array}$ & $6.9(0 \%)$ \\
\cline { 2 - 2 } $\begin{array}{ll}\text { 2 wt\% uniform enrichment } \\
\text { core with gadolinia pin }\end{array}$ & $7.4(0 \%, 40 \%)$ \\
\cline { 2 - 2 } & $6.9(0 \%)$ \\
\hline
\end{tabular}

experiment. The geometrical arrangements of the fuel pin and the alminium tube are illustrated in figure 2.

\section{Benchmark calculation}

The C/E values for $k_{e f f}$ have been calculated for critical experiments using continuous energy Monte Carlo code MVP2 and its libraries generated from ENDF/B-VI.8, ENDF/B-VII.0, JENDL-3.3 and JEFF-3.1. The statistical errors for MVP2 calculation were smaller than $15 \mathrm{pcm}$. Figures 3 , 4, and 5 show the $\mathrm{C} / \mathrm{E}$ values for each core configuration as a function of hydrogen to heavy metal atomic number ratio (H/HM). 


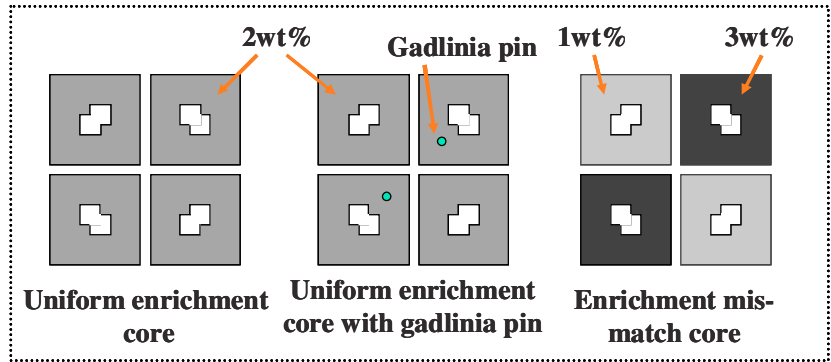

Al-tube arrangement

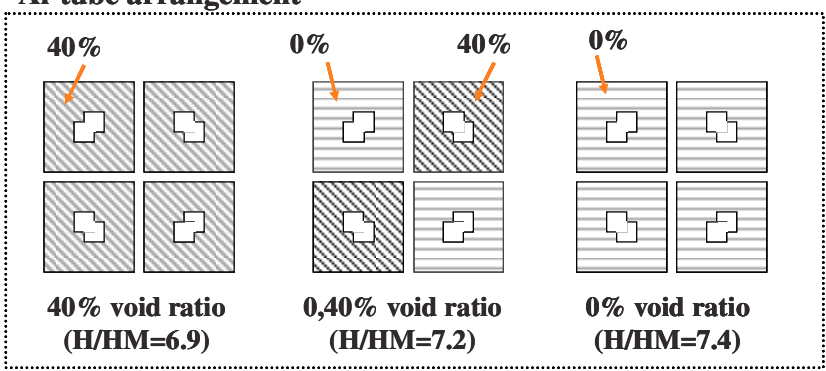

Fig. 2. Fuel pin and Al-tube arrangements of test region.

Deviations of the $\mathrm{C} / \mathrm{E}$ value from unity are $600 \pm 100 \mathrm{pcm}$, $400 \pm 100 \mathrm{pcm}, 200 \pm 100 \mathrm{pcm}$ and $0 \pm 100 \mathrm{pcm}$ for ENDF/BVI.8, JENDL-3.3, JEFF-3.1 and ENDF/B-VII.0, respectively. The agreement of the $\mathrm{C} / \mathrm{E}$ value to unity is excellent for ENDF/B-VII.0, and the long-standing problem of systematic eigenvalue under-prediction in thermal low-enriched uranium fuel rod systems is improved. However, the dependency of the $\mathrm{C} / \mathrm{E}$ on the $\mathrm{H} / \mathrm{HM}$ is still observed for ENDF/B-VII.0. The increment of the $\mathrm{C} / \mathrm{E}$ value from $0 \%$ to $40 \%$ void ratio is about $+50 \mathrm{pcm}$. The existence of the gadolinia pin slightly increases the $\mathrm{C} / \mathrm{E}$ values a few ten $\mathrm{pcm}$ for $\mathrm{ENDF} / \mathrm{B}-\mathrm{VI} .8$, JENDL-3.3 and JEFF-3.1, but no difference was observed for ENDF/VII.0.

\section{Sensitivity analyses}

Sensitivity analyses were performed to specify the source of observed $k_{e f f}$ difference in the nuclear data libraries. The sensitivity is defined by the response of $k_{e f f}$ against the change in nuclear cross-section data. The energy group and reaction-type sensitivities were calculated using TSUNAMI

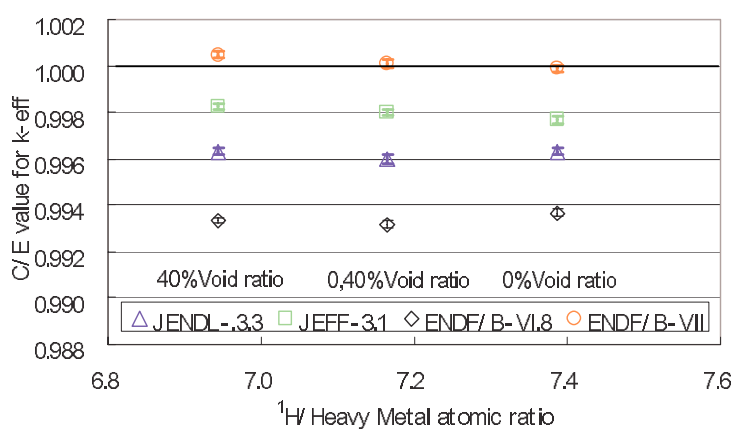

Fig. 3. $\mathrm{C} / \mathrm{E}$ values for $k_{\text {eff }}(2 \mathrm{wt} \%$ of uniform enrichment core).

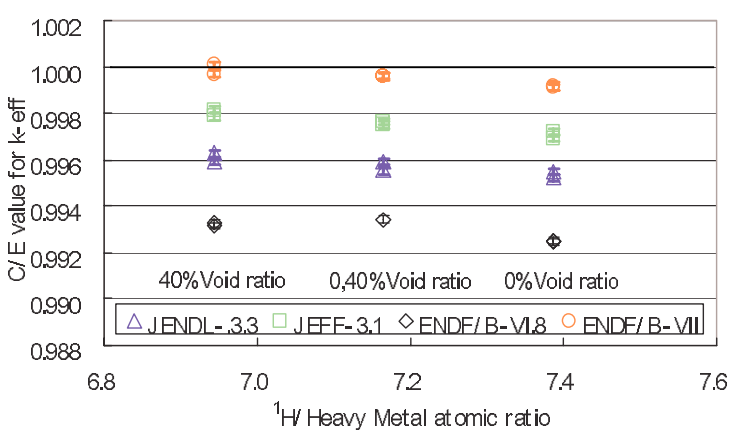

Fig. 4. $\mathrm{C} / \mathrm{E}$ values for $k_{\text {eff }}(1 \mathrm{wt} \%$ and $3 \mathrm{wt} \%$ of enrichment mismatch core).

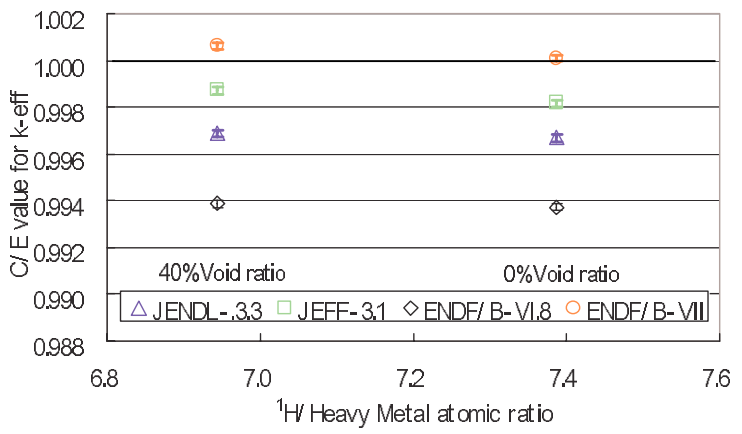

Fig. 5. C/E values for $k_{e f f}(2 \mathrm{wt} \%$ of uniform enrichment core).

sequence [2] in SCALE5.1 package. The TSUNAMI provides the sensitivities with the first-order perturbation theory, which treats the effect of changes in resonance self-shielded crosssections.

In this study, sensitivity analyses were carried out with 2 wt $\%$ enriched $\mathrm{UO}_{2}$ pin cell system with $0 \%$ void ratio. The reactivity contribution to the pin cell $\mathrm{k}_{\mathrm{eff}}$ change of each nuclide and reaction type is evaluated by equation (1) in which $S$ represents sensitivity coefficient for nuclide $n$, for each reaction type $x$ and for each group $g$.

$$
(\delta k / k)_{x}^{n}=\sum_{g} S_{x, g}^{n} \frac{\sigma_{x, g}^{n, l i b 2}-\sigma_{x, g}^{n, l i b 1}}{\sigma_{x, g}^{n, l i b 1}},
$$

where

$(\delta \mathrm{k} / \mathrm{k})_{\mathrm{x}}^{\mathrm{n}}=$ reactivity contribution

$\sigma_{x, g}^{n, l i b 1}=$ micro cross-section in library "lib1".

Figures 6,7 , and 8 show the reactivity contribution to $k_{\text {eff }}$ change from ENDF/B-VI.8 results. Note that the capture reaction in the following figures represents the absorption reaction excluding the fission reaction.

It is clearly observed that the improved $k_{e f f} \mathrm{C} / \mathrm{E}$ values for ENDF/B-VII.0 and JEFF-3.1 are mainly due to a revision in ${ }^{238} \mathrm{U}$ capture cross section. The contributions of this revision to the increased $k_{\text {eff }}$ are about $+0.3 \% \delta \mathrm{k} / \mathrm{k}$. The difference in ${ }^{16} \mathrm{O}$ capture cross section also increase ENDF/B-VII.0 and JENDL-3.3 $k_{\text {eff }}$ compared to ENDF/B-VI.8 about +0.1 and $+0.2 \% \delta \mathrm{k} / \mathrm{k}$ respectively.

The difference of ${ }^{238} \mathrm{U}$ elastic scattering cross-section and $v$-value between JEFF-3.1 and ENDF/B-VI.8 have contribution of about $-0.1 \% \delta \mathrm{k} / \mathrm{k}$ and $+0.1 \% \delta \mathrm{k} / \mathrm{k}$ respectively, which 


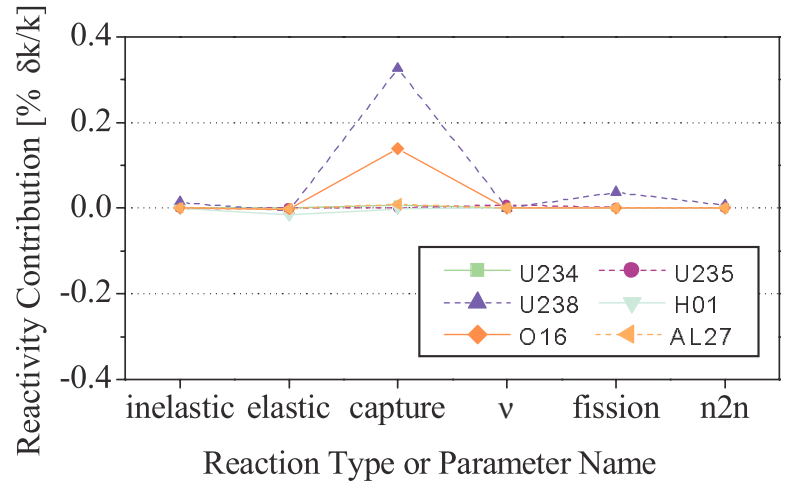

Fig. 6. Reactivity contribution to $k_{e f f}$ change caused by difference of nuclear data between ENDF/VI.8 and ENDF/B-VII.0.

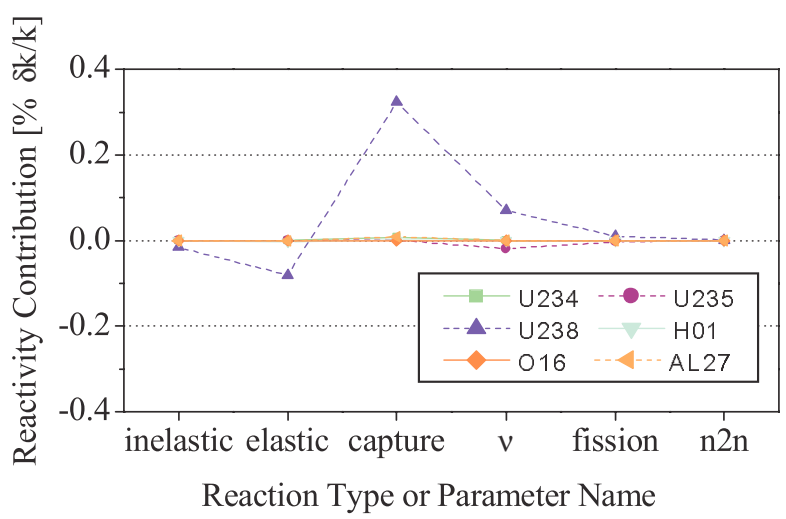

Fig. 7. Reactivity contribution to $k_{\text {eff }}$ change caused by difference of nuclear data between ENDF/VI.8 and JEFF-3.1.

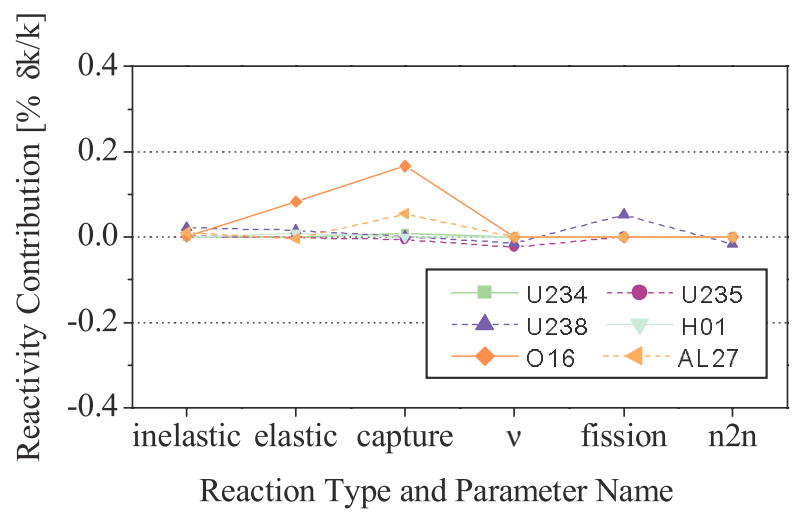

Fig. 8. Reactivity contribution to $k_{\text {eff }}$ change caused by difference of nuclear data between ENDF/VI.8 and JENDL-3.3.

almost cancel out each other. The difference of ${ }^{16} \mathrm{O}$ elastic scattering cross-section between JENDL-3.3 and ENDF/BVI. 8 has contribution of about $+0.1 \% \delta \mathrm{k} / \mathrm{k}$.

Figures 9 and 10 show energy dependency of the sensitivity and contribution to $k_{\text {eff }}$ change from ENDF/B-VI.8 to ENDF/B-VII.0 for ${ }^{238} \mathrm{U}$ capture and ${ }^{16} \mathrm{O}$ capture cross-section. The contribution of ${ }^{238} \mathrm{U}$ capture $+0.3 \% \delta \mathrm{k} / \mathrm{k}$ is caused mainly in thermal and resolved resonance energy range. The magnitude of the contribution due to the thermal range $(<1.0 \mathrm{eV})$ is $+0.17 \% \delta \mathrm{k} / \mathrm{k}$ and resolved resonance range $(1.0 \mathrm{eV}-20 \mathrm{keV})$ is $+0.09 \% \delta \mathrm{k} / \mathrm{k}$. These contributions come from the reduced

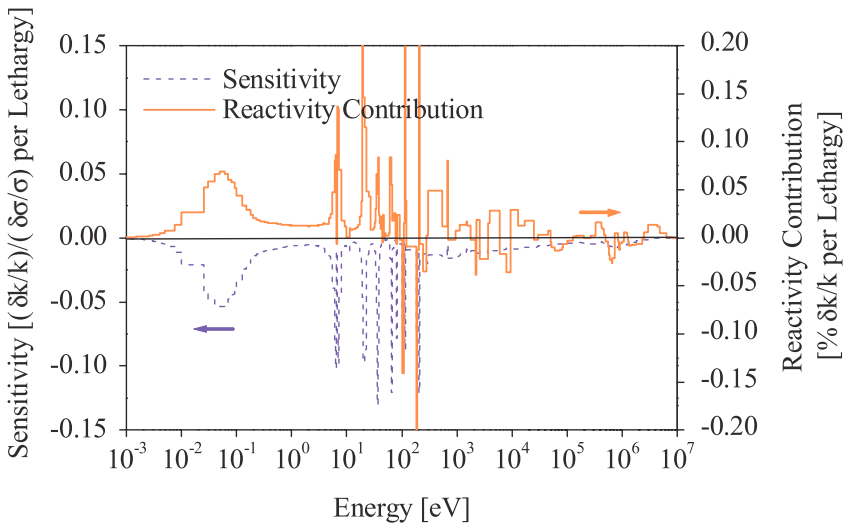

Fig. 9. Energy dependency of ${ }^{238} \mathrm{U}$ capture cross-section sensitivity and contribution caused by difference of nuclear data between ENDF/B-VI.8 and ENDF/B-VII.0.

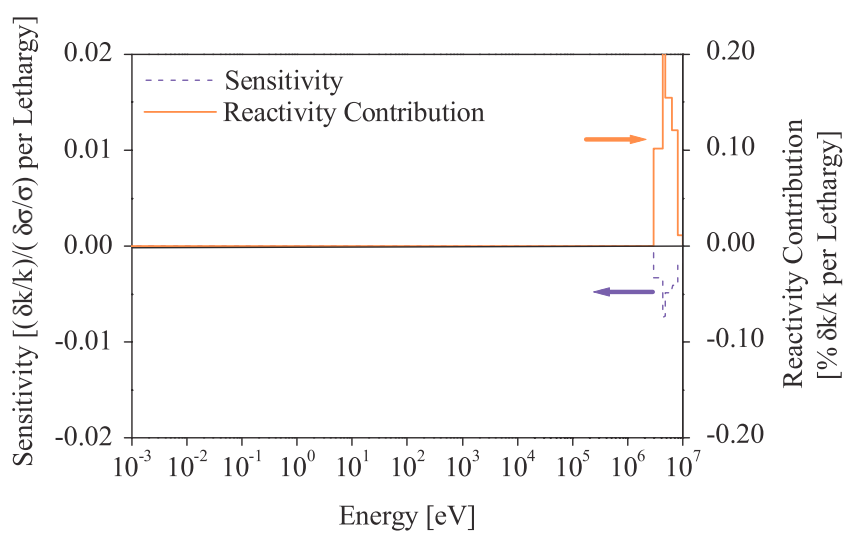

Fig. 10. Energy dependency of ${ }^{16} \mathrm{O}$ capture cross-section sensitivity and contribution caused by difference of nuclear data between ENDF/B-VI.8 and ENDF/B-VII.0.

${ }^{238} \mathrm{U}$ capture cross-section based on the WPEC sub-group-22 recommendation; smaller thermal capture value $2.683 \mathrm{~b}$ at $2200 \mathrm{~m} / \mathrm{s}$ and smaller effective resonance integral [3]. The contribution of ${ }^{16} \mathrm{O}$ is due to the smaller $(\mathrm{n}, \alpha)$ cross-section in the energy range above $3 \mathrm{MeV}$.

\section{Assessment of representativity}

The criticality benchmark testing was performed with critical experiments, in which the moderator densities were set to simulate the BWR hot operating condition. However, they were cold critical experiments under the isothermal room temperature. For discussion of criticality prediction accuracy on the BWR core in hot operating condition, it is important to confirm the representativity of the critical experiments to the applied system. In this study, the representativity is assessed for the $2 \mathrm{wt} \%$ enriched pin cell systems under isothermal room temperature and hot operating thermal condition. The latter is conducted with $784 \mathrm{~K}$ for fuel and clad temperature and $559 \mathrm{~K}$ for moderator temperature.

The representativity is assessed using sensitivity analysis technique, which compare the physical similarity between two systems. A sensitivity-based integral representativity index 
Table 2. $E_{\text {sum }}$ value for the $2 \mathrm{wt} \%$ enriched pin cell systems between isothermal room temperature and hot operating thermal condition.

\begin{tabular}{cc}
\hline Void ratio & $E_{\text {sum }}$ value \\
\hline $0 \%$ & 0.930 \\
$40 \%$ & 0.955 \\
\hline
\end{tabular}

$\mathrm{E}_{\text {sum }}$ is defined by equation 2 , in which $\mathrm{S}_{\mathrm{x}, \mathrm{g}}$ represents sensitivity for each nuclide $n$, for each reaction type $x$, for each group $g$, for the application $a$ and the experiment $e$ [4].

$$
E_{\text {sum }}=\frac{\sum_{x} \sum_{n} \sum_{g}\left(S_{x, g}^{e, n} S_{x, g}^{a, n}\right)}{\sum_{x}\left\{\sum_{n} \sum_{g} S_{x, g}^{e, n} \sum_{n} \sum_{g} S_{x, g}^{a, n}\right\}^{1 / 2}} .
$$

In this definition, the sensitivity data is thought of as a vector, and the integral index $E_{\text {sum }}$ is the cosine of the angle between the two sensitivity vectors for the analyzed systems. Then, the $E_{\text {sum }}$ value of 1.0 indicates the parallel sensitivity vector that represents the complete similarity between two systems. As with the case of an $E_{\text {sum }}$ value of 0.0 indicates orthogonal vector that represents no similarity. The guideline is recommended that an $E_{\text {sum }}$ value of 0.8 or higher indicates enough similarity to the application to be useful in its criticality code validation [5].

The $E_{\text {sum }}$ values for the pin cell systems between isothermal room temperature and hot operating thermal condition are evaluated for $0 \%$ and $40 \%$ void ratio in table 2 . Both of the $E_{\text {sum }}$ values exceed 0.9 which represents enough similarity regardless of the different thermal condition. These results confirmed the effectiveness of the cold critical experiments for the library validation as against the BWR hot operating condition.

\section{Conclusion}

The criticality benchmark testing was performed with evaluated nuclear data libraries ENDF/B-VI.8, ENDF/B-VII.0, JENDL-3.3, and JEFF-3.1 for BWR applications. Deviations of $k_{e f f} \mathrm{C} / \mathrm{E}$ value below unity are $600 \pm 100 \mathrm{pcm}, 400 \pm$
$100 \mathrm{pcm}, 200 \pm 100 \mathrm{pcm}$ and $0 \pm 100 \mathrm{pcm}$ for ENDF/B-VI.8, JENDL-3.3, JEFF-3.1 and ENDF/B-VII.0, respectively. The improvement of criticality prediction is excellent for ENDF/BVII.0, and the long-standing problem of systematic eigenvalue under-prediction in thermal low-enriched uranium fuel rod systems is improved. The dependency of $k_{e f f} \mathrm{C} / \mathrm{E}$ on the $\mathrm{H} / \mathrm{HM}$ is still observed for ENDF/B-VII.0. The increment of $\mathrm{C} / \mathrm{E}$ value from $0 \%$ to $40 \%$ void ratio is about $+50 \mathrm{pcm}$. The existence of gadolinia pin slightly increases the $\mathrm{C} / \mathrm{E}$ values a few ten pcm for ENDF/B-VI.8, JENDL-3.3 and JEFF-3.1, but no difference was observed for ENDF/B-VII.0.

The sensitivity analyses of pin cell system reveal that the primal contribution to increase in keff for ENDF/B-VII.0 and JEFF-3.1 compared to ENDF/B-VI.8 is due to the revision in ${ }^{238} \mathrm{U}$ capture cross-section. The magnitude of the contribution due to the thermal range is about $+0.17 \% \delta \mathrm{k} / \mathrm{k}$ and resolved resonance range is about $+0.09 \% \delta \mathrm{k} / \mathrm{k}$. The difference in ${ }^{16} \mathrm{O}(\mathrm{n}, \alpha)$ cross-section above $3 \mathrm{MeV}$ also increase ENDF/BVII.0 and JENDL-3.3 $k_{\text {eff }}$ compared to ENDF/B-VI.8 by about +0.1 and $+0.2 \% \delta \mathrm{k} / \mathrm{k}$, respectively.

The representativity of cold critical experiments to the BWR hot operating condition is assessed with pin cell calculation using the sensitivity-based integral index $E_{\text {sum }}$. The evaluated $E_{\text {sum }}$ values exceed 0.9 which represents the effectiveness of the cold critical experiments for the library validation as against the BWR hot operating condition.

\section{References}

1. M.B. Chadwick et al., ENDF/B-VII.O: Next Generation Evaluated Nuclear Data Library for Nuclear Science and Technology, Nucl. Data Sheets (2006).

2. B.L. Broadhead et al., Foundations for Sensitivity-Based Criticality Validation Techniques, Trans. Am. Nucl. Soc. 83, 93 (2000).

3. A. Courcelle, First conclusions of the WPEC/Subgroup-22 Nuclear data for improved LEU-LWR reactivity predictions, AIP Conference Proceedings (2004).

4. B.T. Rearden, TSUNAMI Utility Modules, ORNL/TM 2005/39(2006).

5. B.L. Broadhead et al., Sensitivity and Uncertainty Analyses Applied to Criticality Safety Validation, NUREG/CR-6655 (1999). 